\title{
Application of t-Shuffle Permutation Matrices in Delta Interconnection Networks
}

\author{
I. Ž. Milovanović, E. I. Milovanović, M. K. Stojčev, E. Glogić, E. Ć. Dolićanin
}

\begin{abstract}
We define some important properties of the permutation matrices of t-shuffle type which can be used to synthesize omega and inverse omega multistage interconnection networks. We also address some features of these networks compared to other networks of delta type. Finally, we propose one modification of omega network which decreases the number of stages in the network
\end{abstract}

Keywords: Permutation matrices, multistage interconnection networks, delta networks.

\section{Introduction}

Communication subsystems play a very significant role in the today's parallel computers. These subsystems are used to interconnect various processors, memories, disks and other peripherals. The specific requirements of these communications subsystems depend on the architecture of the parallel computer. Multistage Interconnection Networks (MINs) are widely used in parallel multiprocessors systems. Their popularity is due to high switching cost of crossbar networks. Multistage interconnection networks are constructed from cascaded stages to divide the task of permutation in the network into several sub-tasks of lower complexity. A connection pattern between two adjacent stages can be described by a permutation matrix. Therefore these networks are also called permutation interconnection networks.

A binary matrix is a matrix with elements from the set $\{0,1\}$. One important class of these matrices are permutation matrices. A permutation matrix is a square matrix with all elements either 0 or 1 , with exactly one 1 at each row and column. These matrices are used for solving various problems in graph theory [1,2], discrete mathematics [3,4], numerical analysis [5], cryptography [6], etc. Permutation matrices are used to characterize the topology of multistage interconnection networks $[7,8]$.

Manuscript received June 10, 2013; accepted September 3, 2013.

I. Ž. Milovanović, E. I. Milovanović, M. K. Stojčev are with the Faculty of Electrical Engineering, Belgrade, Serbia; E. Glogić and E.Ć. Dolićanin are withthe State University of Novi Pazar, Novi Pazar, Serbia; 
Here we are interested in a special subclass of permutation matrices referred to as $t$ shuffle matrices (see for example [7]). We discuss some properties of these matrices that are of interest for the definition of delta MINs. Delta networks form a class of interconnection networks which includes omega network, butterfly network, binary n-cube, baseline and reverse baseline networks which have all been proven to be topologically equivalent. A delta network of major interest is the network in which the shuffle between each stage is identical.

\section{T-shuffle permutation matrices}

For each given natural number $n, n \geq 3$, and fixed natural number $t, 2 \leq t \leq\left[\frac{n+1}{2}\right]$, one can generate $n$ unity vectors, $\vec{e}_{n, i, j}^{T}$, in the following way (see for example $[7,8]$ )

$$
\begin{gathered}
j:=1,2, \ldots, n \\
i:=1,2, \ldots, t \\
k:=0,1, \ldots,\left[\frac{n-i}{t}\right] \\
\quad \vec{e}_{n, i+k t, j}^{T} ;
\end{gathered}
$$

where $n$ denotes vector length, $i$ location of unity element in the vector, while $j$ corresponds to the generation number. From these vectors a permutation matrix

$$
L_{n}^{t}=\left[\begin{array}{llll}
\vec{e}_{n, i+k t, 1}^{T} & \vec{e}_{n, i+k t, 2}^{T} & \cdots & \vec{e}_{n, i+k t, n}^{T}
\end{array}\right]^{T}
$$

called permutation matrix of t-shuffle, is obtained. When $t=\left[\frac{n+1}{2}\right]$, perfect shuffle permutation matrix is obtained.

By multiplying source vector with a permutation matrix a new vector with rearranged (i.e. permuted) elements with respect to the staring one is obtained. Type of permutation depends on parameter $t$. For example, for $\mathrm{n}=5, \mathrm{t}=2$ and $\mathrm{t}=3$, and starting vector $\vec{x}=\left[\begin{array}{lllll}0 & 1 & 2 & 3 & 4\end{array}\right]^{T}$ we obtain the following permutations of vector $\vec{x}$

$$
\begin{gathered}
L_{5}^{2}=\left[\begin{array}{lllll}
1 & 0 & 0 & 0 & 0 \\
0 & 0 & 1 & 0 & 0 \\
0 & 0 & 0 & 0 & 1 \\
0 & 1 & 0 & 0 & 0 \\
0 & 0 & 0 & 1 & 0
\end{array}\right], \quad\left(\begin{array}{l}
0 \\
1 \\
2 \\
3 \\
4
\end{array}\right) \stackrel{L_{5}^{2}}{\longrightarrow}\left(\begin{array}{l}
0 \\
2 \\
4 \\
1 \\
3
\end{array}\right) \\
L_{5}^{3}=\left[\begin{array}{lllll}
1 & 0 & 0 & 0 & 0 \\
0 & 0 & 0 & 1 & 0 \\
0 & 1 & 0 & 0 & 0 \\
0 & 0 & 0 & 0 & 1 \\
0 & 0 & 1 & 0 & 0
\end{array}\right], \quad\left(\begin{array}{l}
0 \\
1 \\
2 \\
3 \\
4
\end{array}\right) \stackrel{L_{5}^{3}}{\longrightarrow}\left(\begin{array}{l}
0 \\
3 \\
1 \\
4 \\
2
\end{array}\right) .
\end{gathered}
$$


For the given natural number $n, n \geq 3$, and some fixed $t \in N, 2 \leq t \leq\left[\frac{n+1}{2}\right]$, a corresponding t-shuffle permutation matrix has the property that there exists a minimal natural number $r, r \geq 2$ so that the following is valid

$$
\left(L_{n}^{t}\right)^{r} \cdot \vec{x}=L_{n}^{t}\left(L_{n}^{t}\left(\ldots L_{n}^{t}(\vec{x})\right) \ldots\right)=\vec{x} \Leftrightarrow\left(L_{n}^{t}\right)^{r}=I_{n},
$$

where $I_{n}$ is a unity matrix of order $n \times n$. When $t=\left[\frac{n+1}{2}\right]$ or $\mathrm{t}=2$, since $L_{n}^{t}=\left(L_{n}^{2}\right)^{T}$, parameter $r$ is the minimal positive solution of the following modular equation

$$
2^{k} \equiv 1 \bmod \bar{n}, \quad \bar{n}=\left\{\begin{array}{ll}
n-1, & \text { if } n \text { is even } \\
n, & \text { if } n \text { is odd }
\end{array} .\right.
$$

Note that if $n=2^{p}$ or $n=2^{p}-1, p \in N$, then $r=p$. If $n$ is within the range $2^{k-1}+1 \leq$ $n \leq 2^{k}-2$, and $r$ is solution of equation (2), then $r>k$.

\section{Multistage interconnection networks}

Much of the early work on interconnection networks (IN) was motivated by the needs of the communications industry, particularly in the context of telephone switching. With the growth of the computer industry a lot of research effort has been placed on interconnection networks used in multiprocessor architectures for connecting processors to processors and/or memory modules [7].

Multistage interconnection networks are constructed from cascaded stages to divide the task of permutation in the network into several sub-tasks of lower complexity. Each stage consists of a basic switching element (SE) with $a$ inputs and $b$ outputs, denoted as $a \times b$, that represents itself a small crossbar network [9]. Since the complexity of the SE grows with the number of inputs and outputs, SEs are usually implemented as $2 \times 2$ modules. The other two sizes of SEs that are used in practice are $4 \times 4$ and $8 \times 8$. SE can connect any of its inputs to any of its outputs with the restriction that two or more inputs cannot connect to the same output at the same time. Otherwise a conflict occurs. In the case of conflict, one of the requests is passed while the others wait to the next cycle. The outputs of the SEs of each stage are routed to the inputs of the next stage SEs through some inter stage wiring pattern that defines the network topology $[10,11]$. These networks are also called permutation interconnection networks since they can realize all $\mathrm{N}$ ! permutations, where $\mathrm{N}$ is the number of inputs and outputs to the network. Permutations can be represented in several different ways. One often used method is based on permutation matrices.

Various topologies of MINs have been proposed in the last few decades. Many of these topologies are derived from the well known undirected graph topology of the cube network. Networks with a unique path between every input/output pair are called Banyan networks. Delta networks form a subset of Banyan networks [11]. Every Delta network is a Banyan network, but not vice versa. Delta networks have a routing property called self routing. 
This property allows the routing decision to be made by the destination address, regardless of the source address.

\section{Delta MINS}

There may exist large number of link patterns available for delta networks. It should be noted that the probability of acceptance or blocking of messages is identical in all delta networks. This means that one type Delta network can be replaced by another one [9]. Here we will consider delta networks with $2 \times 2$ switching elements.

Let $n=2^{r}, r \geq 1$, be a positive integer and $\vec{x}$ a given vector of length $n$. Under this condition, permutation matrices $L_{1}, L_{2}, \ldots, L_{r}$ exist, so that

$$
L_{1}\left(L_{2}\left(\ldots L_{r} \vec{x}\right)\right)=\vec{x}
$$

and $L_{1} \cdot L_{2} \cdots L_{k} \neq I_{n}$, for all $k$ in the range $1 \leq k \leq r-1$. By the selection of various r-touples of permutation matrices in (3) all possible delta networks can be obtained. (see for example $[10,11])$. The most common are Baseline, Butterfly, Generalized-cube, Omega, etc. There is a wide variety of practical applications where the realizations of data interconnections of these networks are needed, such as FFT and other orthogonal transformations [4 ].

For example, for $r=3, n=2^{3}=8, \vec{x}=\left[\begin{array}{lllllll}0 & 1 & 2 & 3 & 4 & 5 & 6\end{array}\right]^{T}$ and permutation matrices

$$
\begin{aligned}
L_{3} & =\left[\begin{array}{llllllll}
1 & 0 & 0 & 0 & 0 & 0 & 0 & 0 \\
0 & 0 & 0 & 0 & 1 & 0 & 0 & 0 \\
0 & 1 & 0 & 0 & 0 & 0 & 0 & 0 \\
0 & 0 & 0 & 0 & 0 & 1 & 0 & 0 \\
0 & 0 & 1 & 0 & 0 & 0 & 0 & 0 \\
0 & 0 & 0 & 0 & 0 & 0 & 1 & 0 \\
0 & 0 & 0 & 1 & 0 & 0 & 0 & 0 \\
0 & 0 & 0 & 0 & 0 & 0 & 0 & 1
\end{array}\right], \\
L_{2} & =\left[\begin{array}{llllllll}
1 & 0 & 0 & 0 & 0 & 0 & 0 & 0 \\
0 & 0 & 0 & 0 & 1 & 0 & 0 & 0 \\
0 & 0 & 1 & 0 & 0 & 0 & 0 & 0 \\
0 & 0 & 0 & 0 & 0 & 0 & 1 & 0 \\
0 & 1 & 0 & 0 & 0 & 0 & 0 & 0 \\
0 & 0 & 0 & 0 & 0 & 1 & 0 & 0 \\
0 & 0 & 0 & 1 & 0 & 0 & 0 & 0 \\
0 & 0 & 0 & 0 & 0 & 0 & 0 & 1
\end{array}\right]
\end{aligned}
$$




$$
L_{1}=\left[\begin{array}{llllllll}
1 & 0 & 0 & 0 & 0 & 0 & 0 & 0 \\
0 & 0 & 1 & 0 & 0 & 0 & 0 & 0 \\
0 & 1 & 0 & 0 & 0 & 0 & 0 & 0 \\
0 & 0 & 0 & 1 & 0 & 0 & 0 & 0 \\
0 & 0 & 0 & 0 & 1 & 0 & 0 & 0 \\
0 & 0 & 0 & 0 & 0 & 0 & 1 & 0 \\
0 & 0 & 0 & 0 & 0 & 1 & 0 & 0 \\
0 & 0 & 0 & 0 & 0 & 0 & 0 & 1
\end{array}\right]
$$

a generalized-cube MIN presented in Fig. 1 is obtained.

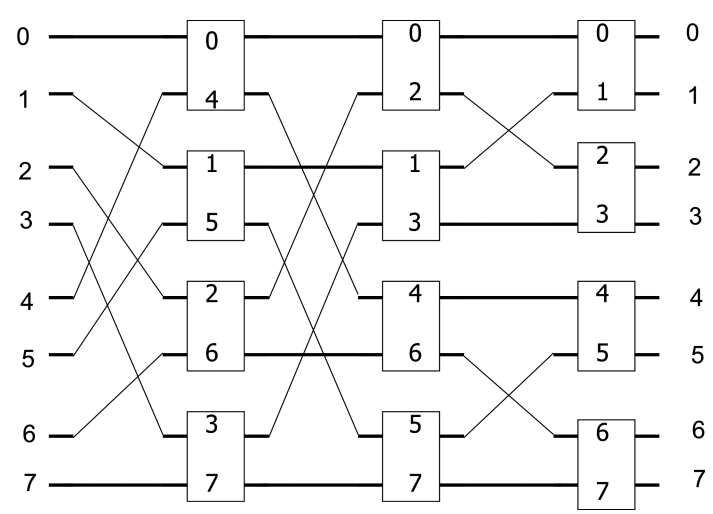

Fig. 1: Generalized cube network for $n=8$.

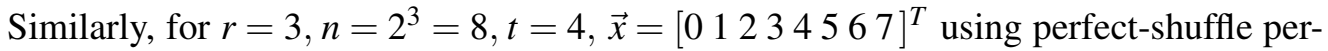
mutation matrix $L_{8}^{4}$, according to equation (1), an Omega network presented in Fig. 2 is obtained.

All delta MINs are proven to be topologically equivalent and can be represented by a graph $G=(V, E)$, where $V=\left\{0,1, \ldots, 2^{r}-1\right\}$ is a set of nodes and $E$ is a set of links. Two nodes $a$ and $b$ in a delta networks are adjacent if they have common SE. Graph $G$ is highly regular, i.e. all nodes have exactly rneighbors. An example of such graph for $r=3$ is represented in Fig. 3.

Definition 1 Let $A\left(a_{i j}\right)$ and $B\left(b_{i j}\right)$ be two arbitrary matrices of order $n \times m$ and $p \times q$, respectively. Tensor product of matrices $A$ and $B$ is denoted with $A \otimes B=\left(a_{i j} B\right)$.

Definition 2 Denote with $I_{n}$ and $\hat{I}_{n}$ a unity matrix and mirror image matrix, both of order $n \times n$. Matrix $I_{n}^{(k \rightarrow)}\left(\right.$ i.e. $\left.\hat{I}_{n}^{(k \rightarrow)}\right)$ is obtained by cyclic shifting of columns of matrix $I_{n}\left(\right.$ i.e. $\left.\hat{I}_{n}\right)$ for $k$ positions to the right.

Adjacency matrix of graph $G=(V, E)$ that corresponds to $r$-dimensional cube network 


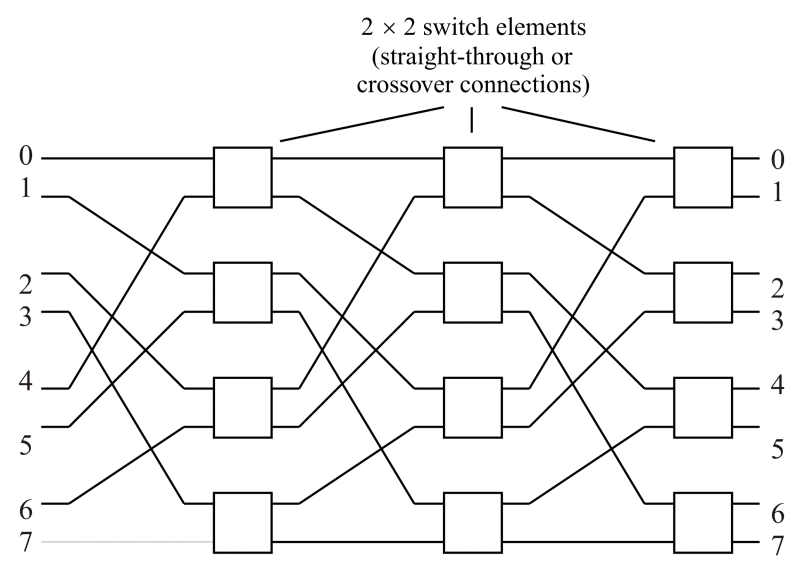

Fig. 2: Omega network for $t=4$ and $n=8$.

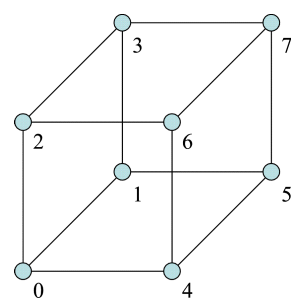

Fig. 3: Cube network for $r=3, n=8$.

can be obtained according to the following recurrences

$$
\begin{aligned}
& M_{2^{2}}=I_{2^{2}}^{(2 \rightarrow)}+\hat{I}_{2^{2}}^{(2 \rightarrow)} \\
& M_{2^{k}}=I_{2} \otimes M_{2^{k-1}}+\hat{I}_{2} \otimes I_{2^{k-1}}
\end{aligned}
$$

for $k=3,4, \ldots, r$.

The spectrum of adjacency matrix $M_{2^{r}}$ is represented by the zeros of characteristic polynomial [1]

$$
f_{2^{r}}(\lambda)=\prod_{i=0}^{r}(\lambda-r+2 i)\left(\begin{array}{c}
r \\
i
\end{array}\right)
$$

From adjacency matrix $M_{2^{r}}$, i.e. its spectrum, some important properties if graph $G=$ $(V, E)$, and consequently of delta networks, can be concluded, such as:

- any node can be reached from any node of the graph;

- there is an unique path from each input node to each output node; 
In general the interconnection pattern between each stage of the delta networks can be different. The most interesting of the delta networks are Omega and Reverse (or Inverse) omega. These networks have a uniform connection pattern between stages, i.e. the same permutation matrix is used to describe all interconnection patterns. In the case of Omega network we have that

$$
L_{1}=L_{2}=\cdots=L_{r}=L_{2^{r}}^{2^{r-1}},
$$

while for the Inverse omega we have

$$
L_{1}=L_{2}=\cdots=L_{r}=L_{2^{r}}^{2}
$$

This is a desirable property since implementation regularity and reusability can greatly reduce the design cost of the MIN. An interesting question is: whether there are other delta MINs with this property?

\section{One modification of Omega MIN}

Delta MINs are blocking networks, meaning that a connection between input/output pair is not always possible because of conflicts with existing connections. To decrease a number of conflicts and increase the throughput of delta MINs, an extra stages can be added or SEs of higher degree can be used. In this way multiple paths between each input/output pair can be obtained and if a conflict occurs on one path the other ones can be used. However this approach increases a communication delay (i.e. number of hops between input and output). The other possibility is to merge switches from various stages into one stage while structure of the first and the last stages remains intact. This structure reduces the number of hops between the source an destination, and thus reduce the probability of blocking [12]. However, the complexity of inner SEs is increased.

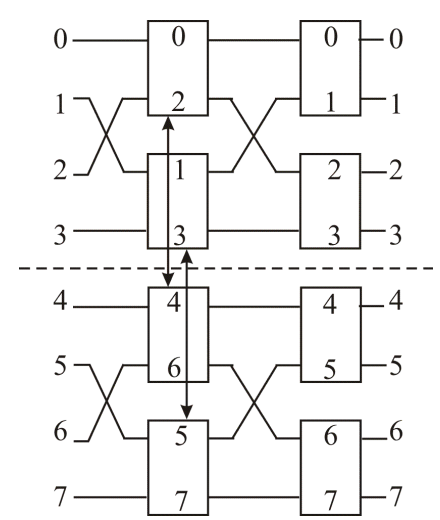

Fig. 4: Modified Omega network 
We propose a modification of omega network that decreases the number of stages for one and preserves the self-routing property of omega network. The idea is as follows. Denote with $0,1,2, \ldots, 2^{r}-1$ inputs (outputs) to the omega MIN. Partition all inputs (outputs) to the network into two groups, such that first group contains inputs (outputs) enumerated by $0,1, \ldots, 2^{r-1}-1$ and the second one with $2^{r-1}, 2^{r-1}+1, \ldots, 2^{r}-1$. Now we construct two omega networks with $r-1$ stages by applying permutation matrix $L_{2^{r-1}}^{2^{r-2}}(r-1)$-times. In order to obtain a path from any input to any output, a two-way connection between $i$ th and $\left(i+2^{r-2}\right)$-th switching elements is established, for $i=1,2, \ldots, 2^{r-2}$. The idea is pictorially represented in Fig. 4 for the case $n=2^{3}$, i.e. $r=3$.

\section{Conclusion}

The systematic method for designing t-shuffle permutation matrices was considered in this paper. Application of these matrices was illustrated on the example of generation of delta multistage interconnection networks. We have also proposed one modification of omega network that can reduce the number of stages in the network, and consequently decrease communication delay while preserving self-routing property of the network.

\section{Acknowledgement}

This research was supported by the Serbian Ministry of Education and Science, under grant TR32009.

\section{References}

[1] A. E. Brouwer, W. H. Haemers Spectra of Graphs, Springer, 2011.

[2] D. CVetKović, P. Rowlinson, S. Simić An introduction to the theory of graph spectra, Cambridge Univ. Press, Cambridge, 2010.

[3] I. Ž. Milovanović, E. I. Milovanović Discrete mathematics, Univ. Niš, 2000. (In Serbian)

[4] E. J. Dagman, G. A. Kuharev, Fast discrete orthogonal transformations, Nauka, Novosibirsk, 1983 (In Russian)

[5] G. V. Milovanović, Numerical analysis, Nauna knjiga, Beograd, 1988. (In Serbian)

[6] A. Dujella, M. Maretic, Cryptography, Element, Zagreb, Zagreb, 2007. (In Croatian).

[7] T. JARVINEN, Systematic methods for designing stride permutation interconnections, Tampere Univ. Tehnol. Tampere, Finland, 2004.

[8] T. Jarvinen, P. SAlmela, H. Sorokin, J. TAKala, Stride permutation network for array processors, J (of VLSI) Signal Processing, Vol. 49, (2007), 51-71. 
[9] I.-R. QUADRI, P. BOUlET, J.L. DEKEYSER, Modeling of topologies of interconnection networks based on multidimensional multiplicity, Research Report, INRIA Futures, May 2007, ISSN 0249-6399.

[10] W. J. Dally, B. Towlesm: Principles and practices of interconnection networks, Morgan Kaufman, San Francisco, CA, 2004.

[11] J. H. PATEL, Performance of processor-memory interconnections for multiprocessors, Proc. $6^{\text {th }}$ Annual Symposium of Computer Arch. New York, 1979, 168-177.

[12] M. MoAZEZ, F. SAFAei, M. RAZAZADEH, Design and implementation of multistage interconnection networks, Int. J. Comput. Sci. Engin. Technol., Vol. 2, 5 (2012), 1-11. 